
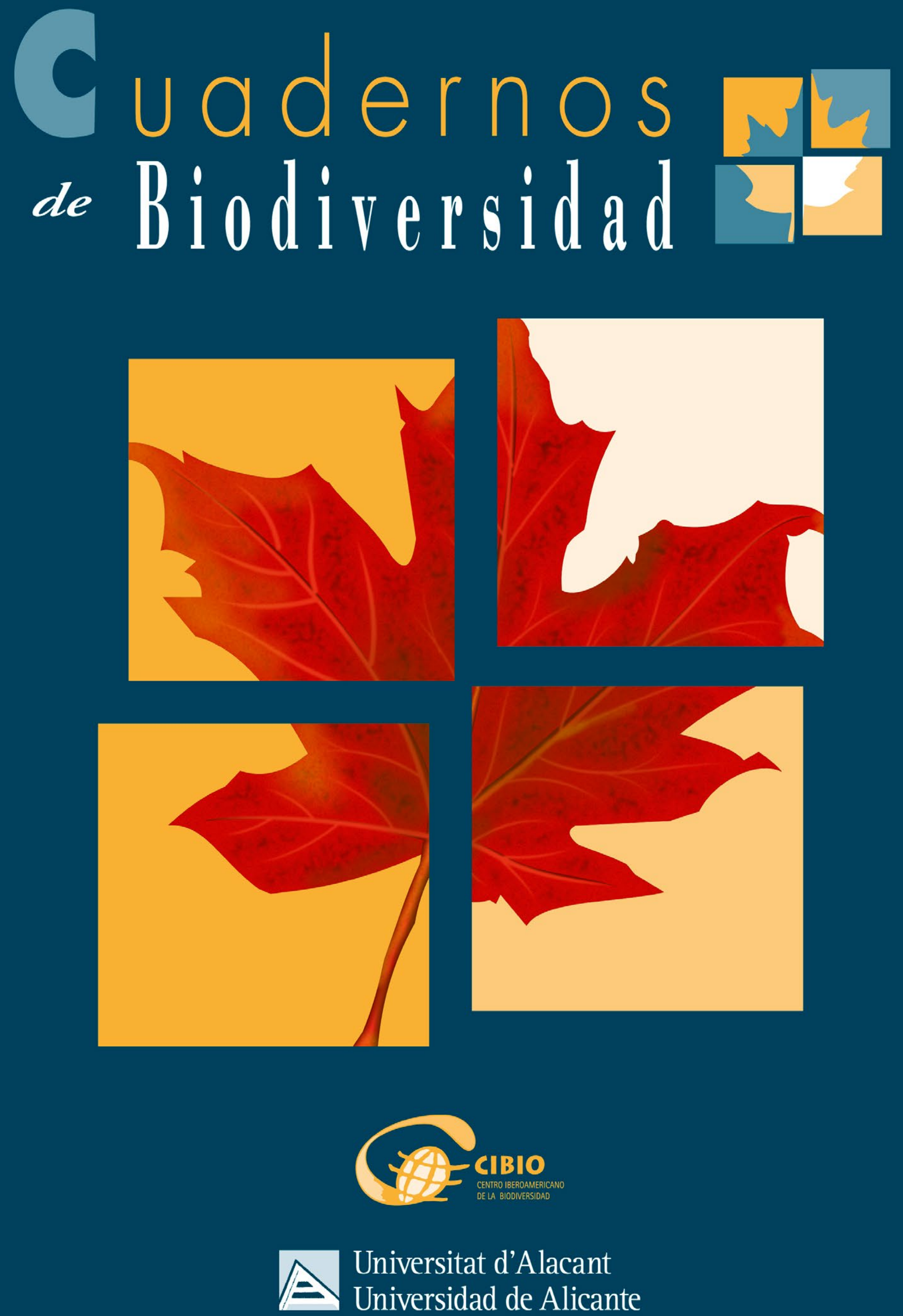


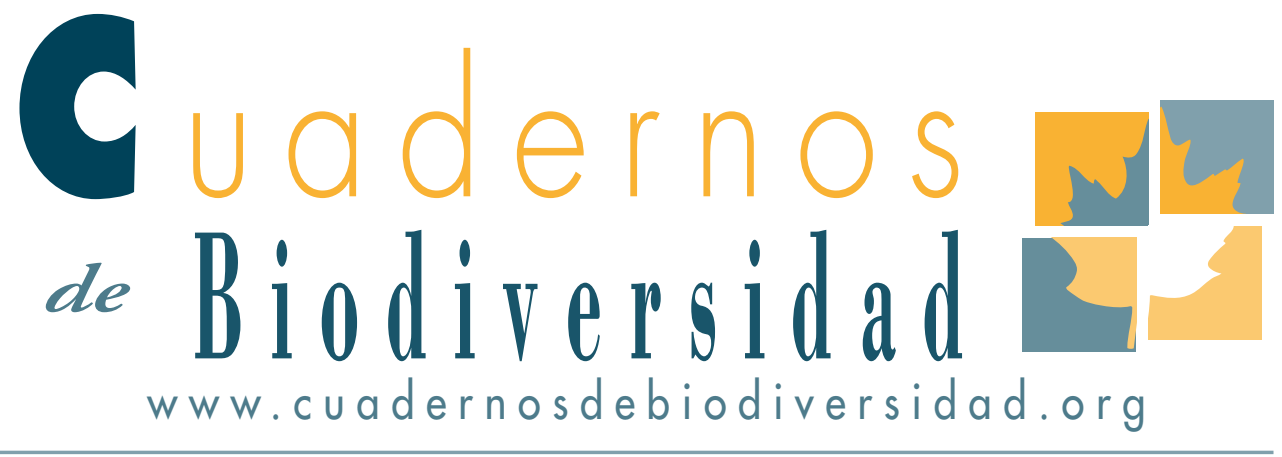

\section{Descripción de una especie nueva del género Athous Eschscholtz, 1829, de la Península lbérica (Coleoptera: Elateridae: Dendrometrinae: Dendrometrini)}

\section{José Luis Zapata de la Vega', Antonio Sánchez-Ruiz²}

i Azafrán, 25. E-28760 Tres Cantos, Madrid (España) Jlzvega@gmail.com

2 Médico Solana, 8-B. E-o26io El Bonillo, Albacete (España) a.S.r@Wanadoo.es

urn:lsid:zoobank.org:pub:F54ADB3B-5F4A-4560-8259-DC838DD22603

\section{RESUMEN}

Se describe una especie nueva de Athous Eschscholtz, 1829 del subgénero Haplathous Reitter, 1905 de la península ibérica: $A$. (H.) dianae sp.n. Esta especie se ha encontrado hasta el momento únicamente en la provincia de Alicante, siendo la primera especie del subgénero Haplathous conocida para la Comunidad Valenciana.

Pallabras clave: Coleoptera, Elateridae, Athous, Haplathous, especie nueva, Taxonomía, Faunística, Península Ibérica.

\section{ABSTRACT}

A new species of the genus Athous Escscholtz, 1829 subgenus Haplathous Reitter, 1905 is described from the Iberian Peninsula: Athous (Haplatous) dianae n.sp. At present this species is only known from the Alicante province, being the first species of Haplathous subgenus known form Valencian Community.

Key words: Coleoptera, Elateridae, Athous, Haplathous, new species, Taxonomy, Chorology, Iberian Peninsula. 


\section{INTRODUCCIÓN}

Dentro de la familia Elateridae Leach, 1815 el género Athous Eschscholtz, 1829 es el que cuenta en la actualidad con más especies conocidas en la península ibérica, con un total de 83. De ellas 9 pertenecen al subgénero Haplathous Reitter, 1905 (Zapata y Sánchez-Ruiz, 2016), que se caracteriza sobre todo por tener el escutelo plano o apenas convexo, truncado en su lado basal y ocupando prácticamente todo el espacio interelitral, por tener los tarsómeros de tamaño decreciente, y por presentar el borde anterior del prosternón con mentonera redondeada en arco amplio (Leseigneur, 1972; Platia, 1994; Laibner, 2000).

El material estudiado de esta nueva especie procede de los muestreos desarrollados en el Parque Natural de la Font Roja, provincia de Alicante, por investigadores del instituto CIBIO de la Universidad de Alicante y en marco, del proyecto Prometeo/ 2013/-034 (financiado por la Generalitat Valenciana).

\section{MATERIAL Y MÉTODOS}

Las medidas para establecer las relaciones morfométricas han sido las siguientes:

- Las antenas: longitud de los artejos medida a lo largo de la línea media, y su anchura la máxima en la perpendicular a ésta.

- Talla medida desde el borde frontal hasta el ápice elitral.

- Longitud del tórax medida a lo largo de la línea media, y su anchura en la perpendicular a ésta, no en los ángulos posteriores, sino en la zona más ancha.

- En los élitros la longitud se ha determinado como la medida desde la base del mismo hasta el ápice y la anchura, máxima dimensión, perpendicular a la longitud.

Se han utilizado las relaciones entre la longitud del pronoto y su anchura LP/AP, y entre la longitud de los élitros respecto a la del pronoto y a la anchura de éstos LE/LP y LE/AE respectivamente.

El material estudiado se encuentra depositado en el Museo Nacional de Ciencias Naturales de Madrid con el No de tipos 2710, en el instituto de investigación CIBIO de la Universidad de Alicante, colección CEUA, y en las colecciones privadas de los autores:

MNCN: Museo Nacional de Ciencias Naturales (Madrid)

CEUA: Colección entomológica de la Universidad de Alicante, Centro Iberoamericano de la Biodiversidad, Universidad de Alicante (Alicante)

JLZ: José Luis Zapata (Madrid)

ASR: Antonio Sánchez-Ruiz (Albacete)

Para la realización de este trabajo se han consultado las siguientes publicaciones con descripciones originales: Brisout (1866), Platia (2008) y Reitter (1904).

\section{RESULTADOS}

\section{Athous (Haplathous) dianae Zapata y Sánchez- Ruiz, sp.n.}

Figuras 1 a 10

Material tipo. Holotipo: España 1ठ Parque Natural Font Roja, Alicante 25.05.2015 Diana Pérez leg. (CIBIO). 24 Paratipos $9 \widehat{\jmath} 15$ ○ 9 , mismos datos que el holotipo salvo la fecha de captura

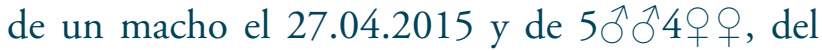
18.05.2016 (MNCN1ðึ1우, CIBIO 6ㅅ11우우, JLZ1ð2우, ASR1ð1우).

\section{Descripción.}

Macho. Enteramente de coloración marrón, sólo los tres primeros antenómeros y patas algo más claras, ocre amarillento; cubierto de una pilosidad densa, corta y tumbada de color blanquecino amarillento (Fig. 1). 


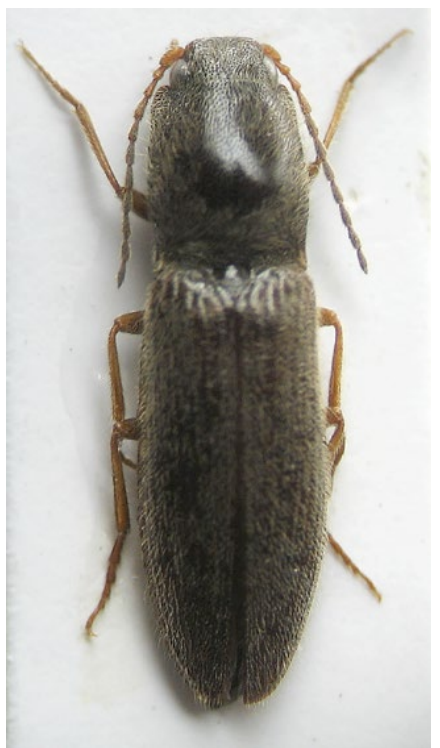

Figura 1: habitus del macho (holotipo)

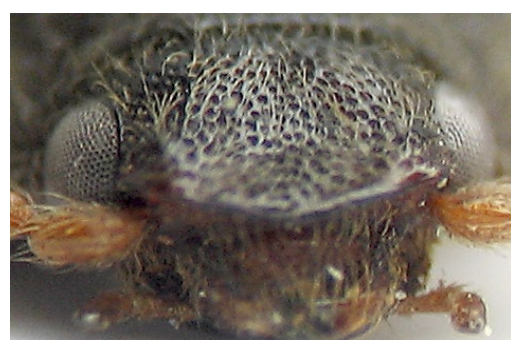

Figura 2: detalle de la frente (paratipo)

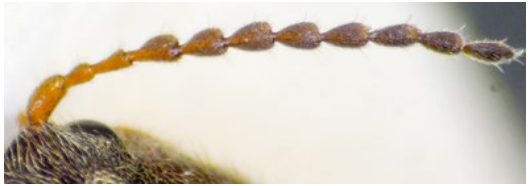

Figura 3: detalle de la antena (paratipo)
Cabeza con punteado intenso, puntos claramente umbilicados muy próximos pero sin llegar a tocarse, separados menos de un diámetro; zona interocular aplanada, muy levemente hundida antes de borde anterior, este grueso y redondeado, algo saliente sobre el epistoma, visto de frente en $\mathrm{V}$ abierta (Fig. 2).

Ojos redondeados, más estrechos que los ángulos anteriores del pronoto. Antenas cortas que apenas superan al pronoto la mitad del último artejo, los tres primeros brillantes y más claros; segundo antenómero cónico más largo que ancho, tercero también cónico casi vez y media más largo que el anterior, el cuarto de longitud inferior a la suma de los dos precedentes juntos, no llega a doblar su anchura, siguientes similares estrechándose progresivamente, undécimo ovalado, de tamaño algo mayor, y longitud casi tres veces su anchura (Fig. 3).

Pronoto levemente convexo, casi rectangular, más largo que ancho, relación LP/AP de 1,11-1,14, con el borde anterior casi recto y con los ángulos anteriores redondeados, avanzando hacia los ojos; lados prácticamente paralelos muy débilmente sinuados antes de los ángulos posteriores, desde el tercio posterior coincidiendo con su máxima anchura; ángulos posteriores cortos y rectos; margen lateral completamente carenado y poco levantado, salvo en el vértice posterior donde se eleva. Punteado marcado, con puntos circulares y umbilicados en los lados, sobre el disco simples, más pequeños y separados un diámetro o más, interespacios lisos. Prosternon acabado en una mentonera arqueada con una amplia escotadura central (Fig. 4).

Escutelo convexo y sobresaliendo de entre los élitros, del mismo color que el pronoto, con pequeños puntos. (Fig. 5)

Élitros subparalelos, 2,41-2,49 veces más largos que el pronoto y 2,36-2,43 su anchura; el máximo de la anchura al superar la mitad; estrías de puntos ovalados alargados, mayores que los puntos de fondo de las interestrías, incisas las cuatro primeras en la parte basal y sólo primera y segunda en la zona apical; interestrías aplanadas a algo convexas, con punteado visible y cierta rizadura transversal.

Edeago como en fig. $6(0,95 \mathrm{~mm})$. Escleritos abdominales asociados a la genitalia según fig. 7 .

Dimensiones. Longitud 7,55-7,80 $\mathrm{mm}$ y anchura $2,2 \mathrm{~mm}$.

Hembra. Muy parecida al macho en su aspecto general y coloración, algo más ancha; pronoto más convexo (LP/AP $=1,07-1,10)$, y ligeramente más grande. Élitros con las siguientes relaciones: LE/PL entre 2,25 y 2,49, oscilando la LE/AE entre 2,16 y 2,24. Antenas más cortas, le faltan dos artejos para alcanzar los ángulos posteriores (Fig. 8). Bolsa copulatriz de morfológia tipo "vittatus". (Fig. 9). 


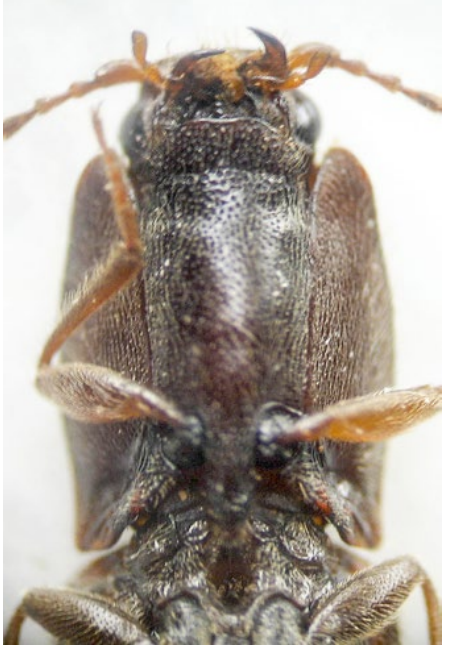

Figura 4: detalle del prosterno (holotipo)

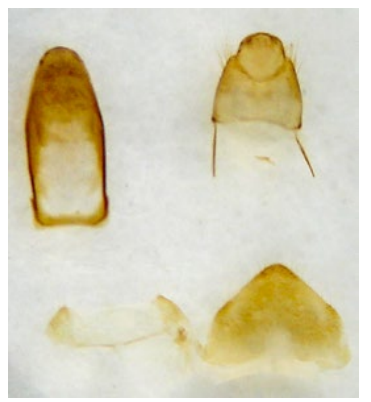

Figura 7: detalle de los terguitos (paratipo)

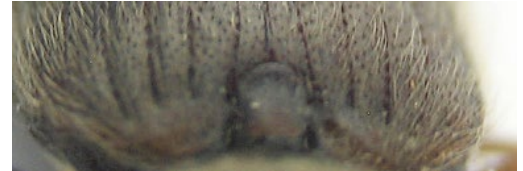

Figura 5: detalle del escutelo (paratipo)

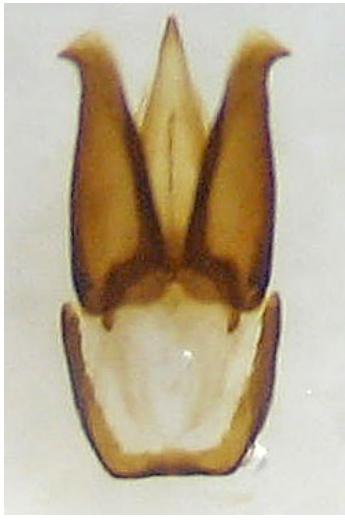

Figura 6: visión dorsal del edeago (holotipo)

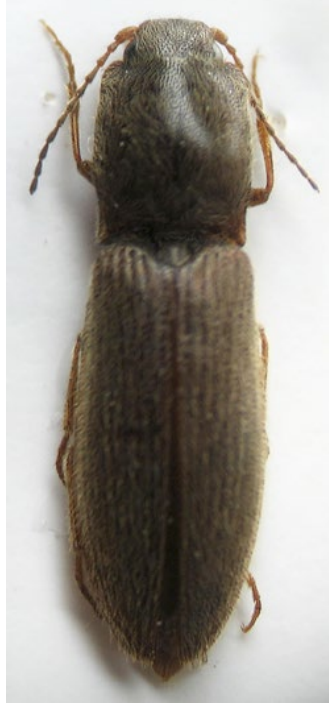

Figura 8: habitus de la hembra (paratipo)

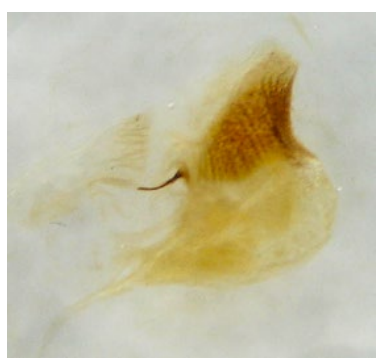

Figura 9: bolsa copulatriz de la hembra (paratipo)

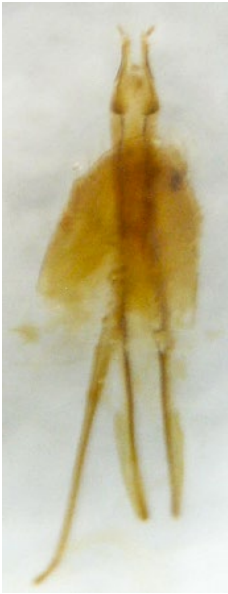

Figura 10: ovopositor (paratipo)
Dimensiones. Longitud $8,4-9,1 \mathrm{~mm}$ y anchura 2,25-2,75mm.

Variabilidad. Muy constante en su coloración y punteado, sólo algún ejemplar presenta leve enrojecimiento en los ángulos posteriores del pronoto; la pilosidad en las hembras pasa del blanco amarillento al amarillo pálido, y en un ejemplar son levemente divergentes sus ángulos posteriores.
Diagnosis. Se diferencia de las especies ibéricas del subgénero Haplathous por su tamaño pequeño, sólo $A$. (H.) reynosae Brisout, 1866 y $A$. (H.) montserratensis Platia, 2008 tienen talla semejante. De la primera es claramente diferenciable por su coloración habitualmente bicolor, con el pronoto marrón oscuro o negruzco, orlado de una banda testácea ferruginosa al igual que los élitros, y las 
antenas alcanzando el escutelo; de la segunda se diferencia igualmente por la longitud de las antenas que superan en dos artejos los ángulos posteriores del pronoto y el tamaño del cuarto tarsómero muy corto respecto al tercero, y en ambos casos por la configuración del edeago: parámeros curvados, lóbulo central más delgado y ápice muy fino en $A$. (H.) montserratensis y diferente de $A$. (H.) reynosae por sus parámeros más cortos y esbeltos que la nueva especie, y sobretodo porque las ramas posteriores del lóbulo central apenas rebasan la base de los parámeros en $A$. (H.) reynosae, cuando lo hacen claramente en $A$. (H.) dianae.

Distribución. España. Únicamente conocida por el momento del entorno correspondiente al Parque Natural de la Font Roja en el término de Alcoy, provincia de Alicante. Constituyendo además no solo el primer representante del subgénero para la provincia sino también para la comunidad valenciana.

Datos ecológicos. El conjunto de ejemplares fueron capturados a una altitud de $1.275 \mathrm{~m}$, con trampas de ventana sobre Quercus rotundifolia, a excepción de un sólo individuo hembra que se recolectó en trampa de emergencia situada en una oquedad también en $Q$. rotundifolia a $1.091 \mathrm{~m}$ junto a dos machos y una hembra que a igual altitud fueron recogidos en trampa de ventana. El tipo de trampas utilizado está descrito en Quinto et al. (2013)

Etimología. La especie está dedicada a Diana Pérez, legataria de la serie típica.

\section{AGRADECIMIENTOS}

Queremos expresar nuestro reconocimiento a Estefanía Micó, que nos ha facilitado los especímenes y al proyecto (PROMETEO/2013/034, I.P. Eduardo Galante) subvencionado por la Generalitat Valenciana

\section{REFERENCIAS}

Brisout, C. (1866) Excursion de 1865, Coléoptères nouveaux trouvés en Espagne pendant l'excursion de la Société en 1865. Annales de la Societé Entomologuque de France, 6: 345-426.

Laibner, S. (2000) Elateridea of the Czech and Slovak Republics. Ed. Kabourek, s.r.o. Zlín. 292 pp.

Leseigneur, L. (1972) Coléoptères Elatérides de la fauna de France continentale et de Corse. Supplément au Bulletin mensuel de la Société Linnéenne de Lyon 41: 1-381.

Platia, G. (1994) Coleoptera, Elateridae. En: Fauna d'Italia. 33, XIV + 429 pp. Calderini Bologna Ed., Bologna.

Platia, G. (2008) Descrizione di un nuovo Athous attero del gruppo corsicus Reiche, della Catalogna (Spagna) (Coleoptera, Elateridae, Dendrometrini). Boletín de la Sociedad Entomológica Aragonesa, 43: 23-24.

Quinto J., Marcos-García MA, Brustel H, Galante E., Micó E. (2013) Effectiveness of three sampling methods to survey saproxylic beetle assemblages in Mediterranean Woodland. Journal of Insect Conservation, 17: 765-776.

Reitter, E. (1904) Neue Arten des Coleopter-Genus Athous Eschsch. Aus Spanien. Boletin de la Real Sociedad Española de Historia Natural, 4: 236-240

Zapata J.L. y Sánchez-Ruiz, A. (2016) Nuevas aportaciones al catálogo de la familia Elateridae (Coleoptera) en la Península Ibérica e Islas Baleares, IV. Arquivos Entomolóxicos, 15: 175-205 